\title{
Antiseptic, Aseptic and Sterilizing Practices in General Dentistry- In the Age of COVID-19: An Appraisal
}

\author{
Louis ZG Touyz ${ }^{*}$ \\ ${ }^{1}$ McGill University Faculty of Dentistry, PQ Canada \\ *Corresponding Author: Louis ZG Touyz, Director and Professor Periodontics, McGill University Faculty of \\ Dentistry, PQ Canada; Email: Touyzlouis@gmail.com
}

Received Date: 26-06-2020; Accepted Date: 17-07-2020; Published Date: 24-07-2020

Copyright $^{\oplus} 2020$ by Touyz LZG. All rights reserved. This is an open access article distributed under the terms of the Creative Commons Attribution License, which permits unrestricted use, distribution, and reproduction in any medium, provided the original author and source are credited.

\begin{abstract}
This appraisal recalls past best antiseptic practices, discusses and underlines the necessity, importance and practice of optimal contemporary infection-control, for dentists in the COVID19 pandemic.
\end{abstract}

\section{Keywords}

Antisepsis; COVID-19; Dentists; Infection; Microbiota; Practitioner; Virus

\section{Introduction}

Few current (2019) general dental practitioners recall those days when antisepsis was relatively primitive compared to today. There was a time up to the mid twentieth century (1900 to 1960) when soap and hot water were considered adequate for asepsis (reducing viable organic organisms to the level of no transmission of infection). Washing hands and scrubbing fingernails under running tepid tap-water with an antiseptic phenol-laced soap and sing "Happy birthday to oneself twice, was considered adequate to ensure manual cleanliness no transfer of microbes. Most general dental practitioners and oral health care workers like oral hygienists, dental assistants and nurses, all practiced with un-gloved hands. Many did not wear face masks. Eye protection was with ordinary spectacles. Exodontics and minor oral surgical procedures were done with naked hands and the likelihood of receiving or transferring any infection was 
considered negligible. Some endodontists claim they could digitally feel when endo-reamers reached the apex. Alginate impressions were kept moist with gauze soaked in tap-water and transported thus to technicians, with no regards to possibilities of infection. Boiling water "sterilizers" were kept boiling all day to "sterilize hand instruments." Each time one opened the lid covering the boiling cauldron, your spectacles would steam up. Dry heat sterilization was kept for special endodontic instruments [at $120^{\circ} \mathrm{C}$ overnight], and most heat labile supports like rubber tubing and bowls, plastic instruments, mixing spatulas, were simply wiped down with surgical ethanol $(90 \% \mathrm{~V} / \mathrm{V})$. These techniques were not reliable and bacterial spores often survived these practices, even though the entire operatory could be wiped down with ethanol or some other antiseptic liquid. Herpes infection of dentists fingers were known, as was an increased prevalence of antibodies to hepatitis viruses among dentists, and also spores of fungi (Candida species) were commonly found under finger nails of dentists. In the mid 1950's the contraceptive pill for females was introduced and then globally in the 1960's much libidinous sexual behaviour followed. In the 1970's the scourge of HIV started to manifest when many young homosexual men between 18 and 28 were dying from AIDS related disease like Pneumocystis corinii. Being infected with HIV, was considered a guaranteed death sentence as most HIV infected patients succumbed to AIDS within a decade of the initial infection. Even though the main method of infection was through sexual intercourse, HIV infection from any and all sources was deemed possible and a patient (Kimberly Bergalis) accused her gay dentist (Dr Acer) of infecting her with HIV. History proved this accusation to be spurious if not false, but the publicity generated in the seventies from this case shocked the entire dental profession world-wide into adopting more stringent disinfection practices. In 2020 the COVID-19 virus pandemic inflicted global populations. Infected individuals without showing any overt signs or symptoms could be unwittingly transmitters of the virus through respiratory droplet spread. Through COVID-19 infected carriers flying internationally, the virus spread throughout the world and has infected millions of people, with mortality rates between 4 and 10 per cent.

\section{Aim}

This subjective appraisal recalls anachronistic practices and underlines the necessity to strive for and maintain improved sterilization practices in general dentistry, and suggests effective accepted alternate therapy modalities in dentistry to minimize chance of transmitting COVID19 in dental operatory.

\section{Current Practice}

The vast majority of licensed general dental practitioners now used proven techniques and practices to sustaining infection control and minimize to the point of extreme, the lowest chances of transmitting or nurturing any infection. These practices are now routine, are taught to aspiring dental students as part of their training, and standards are sustained through periodic 
assessments and monitoring by professional licensing bodies. Placing an ungloved hand into any orifice now is considered an assault, and dentists now not only wash their hands regularly between patients, but also don clean sterile gloves for any examination or intra-oral work to be done. Frequent washing of hands for 30 seconds with an antiseptic soap, before during and after treatment by the dentist and staff is mandatory. Face masks and eye protection is now behind impenetrable masks and transparent eye screens or spectacles. Most practices have mini-autoclaves that successfully sterilize (leaving no viable organic material) using steam or other gases and/or vapours (like ethylene at $120^{\circ} \mathrm{C}$ under 10 kilos of pressure) for all instruments prior to use. Disposable clothing is often used or sterilizing laundry practices used for re-usable items, like covers, towels, cloth screens and gowns. Social distancing between patients is mandatory, and ensuring arriving patient's wash/disinfect their hands upon arrival is also essential.

Cleaning and sterilizing protocols are now part and parcel of contemporary dental practice. The operatory chairs need to be disinfected with wipe-down antiseptic solutions between patients, and also the entire operatory at the end and beginning of each day. This includes the waiting room and other rooms used in the dental office, all door handles, telephones and service counters. Highly effective disinfectant liquids are freshly prepared daily and used to disinfect all furnishings in the operatory. At night many operatories have ultra-violet light burning to ensure surface disinfection. Even sterile disposable over-gowns, head-coverings and foot covers may be used to assist in sustaining a disinfected environment. Impressions are mixed with antiseptic solutions and transported in disinfectant liquids to protect dental technicians.

\section{Discussion}

Many people think the new approaches are "overkill", and add unnecessarily to the high cost of dentistry. Yet since these measures were introduced it is extremely rare that any infections from dental practitioners have ever been reported. Assuming there are some 50,000 registered practicing dentists in North America, and that each sees about 10 patients a day for 200 working days a year, the situations for possibly being infected would be 100,000,000 each year. That not one case of transmission of infection from registered modern practicing dentists has been noted now for decades speaks volumes to the success of the modern approach to infection control in dental operatories.

\section{With Regard to the COVID-19 Virus Transmission}

Most contemporary dental operatories have high speed-drills and ultra-sonic cleaners. Consequently when these are used high speed liquid-evacuation and air-suction are used to minimize consequent spray-spread. Also the operator and support staff wear microbial impenetrable masks and solid transparent plastic face-and neck screens to minimize droplet 
atmospheric spread. Ultra violet light, which is an effective sterilizing agent against surface microbial survival, is used to disinfect hard exteriors where instruments and other clinical paraphernalia may reside when not in use.

High speed aero-turbine-driven drill does markedly facilitate hard tooth-material preparation, with speeds of up to 100,000 rotations per minute. With ultra-sonic vibratory-scalers (like the Cavitron) using an electric-solenoid to induce cleaning ultra-sonic vibrations (at 500,000 or more $\mathrm{Hz}$ ); the heat generated is kept down at source with a cool water spray. Both techniques significantly expedite therapy: yet alternate but much slower and constrained techniques are available for necessary treatments.

1. The use of the electric powered slower speed drill, with rotation at a maximum of $6000 \mathrm{rpm}$, spray-spread is markedly reduced

2. Hand-scaling (removal of extrinsic tooth material, calculus, stain and debris) and rootplaning (removal of intrinsic tooth material, infected and affected by microbes) is an effective, if not preferred alternate treatment modality. Both are effective instruments to use, but take considerably more time.

Most liquid disinfectants like chlorinated bleach, chemo-disinfectants (like Cidex), surgical alcohol (90\% v/v) are used liberally in modern operatories and eliminate nearly all microbes when used.

Crowded waiting rooms, with 2 or more patients, should not be allowed. One patient waiting a short period before an appointed time is tolerated, but two or more people should be avoided, and social distancing ( 2 meters) be sustained in practice.

COVID-19 is an RNA-virus and like most other RNA viruses (Picornaviruses), it can mutate; the newest corona virus is called SARS-CoV-2, and can remain undetected for a long time. Covid-19 and SARS-CoV-2 both are transmitted though droplet spread. Even if symptomless (no fever, dry cough, feeling ill) the viruses can be spread before these symptoms manifest in infected individuals.

Alas, increased effective infection control is demanded, practiced and sustained in most modern dental operatories, but has to be paid for and amortized in dental fees. Public health and professional standards must conform and ensure these disinfection techniques are implemented and carried out. 


\section{Concluding Remarks}

One unnecessary transfer of infection from dentist to patient, or vice versa, with any morbidity or mortality would be one too many. Technology introduces novel ideas regularly to improve the dental profession, but the fundamentals of infection control are prime, and must be rigorously sustained for the health of all dentists, their patients, the community at large, and the whole profession. 